\title{
A reverse Hardy-Hilbert-type integral inequality involving one derivative function
}

\section{Qian Chen ${ }^{1 *}$ and Bicheng Yang ${ }^{2}$}

\section{*Correspondence:}

cq_c123@163.com

${ }^{1}$ Department of Computer Science, Guangdong University of

Education, Guangzhou, Guangdong 51003, China

Full list of author information is available at the end of the article

\begin{abstract}
In this article, by using weight functions, the idea of introducing parameters, the reverse extended Hardy-Hilbert integral inequality and the techniques of real analysis, a reverse Hardy-Hilbert-type integral inequality involving one derivative function and the beta function is obtained. The equivalent statements of the best possible constant factor related to several parameters are considered. The equivalent form, the cases of non-homogeneous kernel and some particular inequalities are also presented.
\end{abstract}

MSC: $26 \mathrm{D} 15$

Keywords: Weight function; Hardy-Hilbert-type integral inequality; Derivative function; Parameter; Beta function; Reverse

\section{Introduction}

Assuming that $0<\sum_{m=1}^{\infty} a_{m}^{2}<\infty$ and $0<\sum_{n=1}^{\infty} b_{n}^{2}<\infty$, we have the following Hilbert inequality with the best possible constant factor $\pi$ (cf. [1], Theorem 315):

$$
\sum_{m=1}^{\infty} \sum_{n=1}^{\infty} \frac{a_{m} b_{n}}{m+n}<\pi\left(\sum_{m=1}^{\infty} a_{m}^{2} \sum_{n=1}^{\infty} b_{n}^{2}\right)^{1 / 2} .
$$

If $0<\int_{0}^{\infty} f^{2}(x) d x<\infty$ and $0<\int_{0}^{\infty} g^{2}(y) d y<\infty$, then we still have the integral analogue of (1) as follows (cf. [1], Theorem 316):

$$
\int_{0}^{\infty} \int_{0}^{\infty} \frac{f(x) g(y)}{x+y} d x d y<\pi\left(\int_{0}^{\infty} f^{2}(x) d x \int_{0}^{\infty} g^{2}(y) d y\right)^{1 / 2}
$$

where the constant factor $\pi$ is the best possible. Inequalities (1) and (2) with their extensions play an important role in analysis and its applications (cf. [2-13]).

The following half-discrete Hilbert-type inequality was presented in 1934 (cf. [1], Theorem 351): If $K(x)(x>0)$ is a non-negative decreasing function, $p>1, \frac{1}{p}+\frac{1}{q}=1,0<\phi(s)=$ $\int_{0}^{\infty} K(x) x^{s-1} d x<\infty, f(x) \geq 0,0<\int_{0}^{\infty} f^{p}(x) d x<\infty$, then

$$
\sum_{n=1}^{\infty} n^{p-2}\left(\int_{0}^{\infty} K(n x) f(x) d x\right)^{p}<\phi^{p}\left(\frac{1}{q}\right) \int_{0}^{\infty} f^{p}(x) d x
$$

(c) The Author(s) 2020. This article is licensed under a Creative Commons Attribution 4.0 International License, which permits use, sharing, adaptation, distribution and reproduction in any medium or format, as long as you give appropriate credit to the original author(s) and the source, provide a link to the Creative Commons licence, and indicate if changes were made. The images or other third party material in this article are included in the article's Creative Commons licence, unless indicated otherwise in a credit line to the material. If material is not included in the article's Creative Commons licence and your intended use is not permitted by statutory regulation or exceeds the permitted use, you will need to obtain permission directly from the copyright holder. To view a copy of this licence, visit http://creativecommons.org/licenses/by/4.0/. 
In recent years, some new extensions and reverses of (3) were presented by [14-19].

In 2006, by using Euler-Maclaurin summation formula, Krnic et al. [20] gave an extension of (1) with the kernel $\frac{1}{(m+n)^{\lambda}}(0<\lambda \leq 14)$. In 2019-2020, using the results of [20], A diyasuren et al. [21] considered an extension of (1) involving the partial sums, and Mo et al. [22] gave an extension of (2) involving the upper limit functions. In 2016-2017, by applying the weight functions, Hong et al. [23, 24] considered some equivalent statements of the extensions of (1) and (2) with several parameters. For some similar work, see [25-28].

In this paper, following [21, 23], by the use of weight functions, the idea of introducing parameters, the reverse extension of (1) and the technique of real analysis, a reverse Hardy-Hilbert-type integral inequality with the kernel $\frac{1}{(x+y)^{\lambda+1}}(\lambda>0)$ involving one derivative function and the beta function is given. The equivalent statements of the best possible constant factor related to several parameters are considered. The equivalent form, the cases of non-homogeneous kernel and a few particular inequalities are obtained.

\section{Some lemmas}

In what follows, we assume that $0<p<1, \frac{1}{p}+\frac{1}{q}=1, \lambda>0, \lambda_{i} \in(0, \lambda)(i=1,2), a:=\lambda-\lambda_{1}-$ $\lambda_{2}, f(x)$ is a non-negative measurable function in $R_{+}=(0, \infty)$, and $g(y)$ is a non-negative increasing differentiable function unless at finite points in $R_{+}$, with $g(y)=o(1)\left(y \rightarrow 0^{+}\right)$, $g(y)=o\left(e^{t y}\right)(t>0 ; y \rightarrow \infty)$ satisfying

$$
0<\int_{0}^{\infty} x^{p\left(1-\lambda_{1}\right)-a-1} f^{p}(x) d x<\infty \quad \text { and } \quad 0<\int_{0}^{\infty} y^{q\left(1-\lambda_{2}\right)-a-1} g^{\prime q}(y) d y<\infty .
$$

By the definition of the gamma function, for $\lambda, x, y>0$, the following expression holds (cf. [29]):

$$
\frac{1}{(x+y)^{\lambda}}=\frac{1}{\Gamma(\lambda)} \int_{0}^{\infty} t^{\lambda-1} e^{-(x+y) t} d t
$$

where the gamma function is defined by

$$
\Gamma(\alpha):=\int_{0}^{\infty} e^{-t} t^{\alpha-1} d t \quad(\alpha>0),
$$

satisfying

$$
\Gamma(\alpha+1)=\alpha \Gamma(\alpha) \quad(\alpha>0) .
$$

Lemma 1 For $t>0$, we have the following expression:

$$
\int_{0}^{\infty} e^{-t y} g(y) d y=\frac{1}{t} \int_{0}^{\infty} e^{-t y} g^{\prime}(y) d y .
$$

Proof Since $g(y)=o(1)\left(y \rightarrow 0^{+}\right)$, we find

$$
\begin{aligned}
\int_{0}^{\infty} e^{-t y} g^{\prime}(y) d y & =\int_{0}^{\infty} e^{-t y} d g(y) \\
& =\left.e^{-t y} g(y)\right|_{0} ^{\infty}-\int_{0}^{\infty} g(y) d e^{-t y}=\lim _{y \rightarrow \infty} \frac{g(y)}{e^{t y}}+t \int_{0}^{\infty} e^{-t y} g(y) d y .
\end{aligned}
$$


In view of $g(y)=o\left(e^{t y}\right)(t>0 ; y \rightarrow \infty)$, we have $\lim _{y \rightarrow \infty} \frac{g(y)}{e^{t y}}=0$, and then

$$
t \int_{0}^{\infty} e^{-t y} g(y) d y=\int_{0}^{\infty} e^{-t y} g^{\prime}(y) d y
$$

namely, Eq. (5) follows.

The lemma is proved.

Lemma 2 Define the following weight functions:

$$
\begin{aligned}
& \varpi\left(\lambda_{2}, x\right):=x^{\lambda-\lambda_{2}} \int_{0}^{\infty} \frac{t^{\lambda_{2}-1}}{(x+t)^{\lambda}} d t \quad\left(x \in \mathrm{R}_{+}\right), \\
& \omega\left(\lambda_{1}, y\right):=y^{\lambda-\lambda_{1}} \int_{0}^{\infty} \frac{t^{\lambda_{1}-1}}{(t+y)^{\lambda}} d t \quad\left(y \in \mathrm{R}_{+}\right) .
\end{aligned}
$$

We have the following expressions:

$$
\begin{array}{ll}
\varpi\left(\lambda_{2}, x\right)=B\left(\lambda_{2}, \lambda-\lambda_{2}\right) & \left(x \in \mathrm{R}_{+}\right), \\
\omega\left(\lambda_{1}, y\right)=B\left(\lambda_{1}, \lambda-\lambda_{1}\right) & \left(y \in \mathrm{R}_{+}\right),
\end{array}
$$

where $B(u, v):=\int_{0}^{\infty} \frac{t^{u-1}}{(1+t)^{u+v}} d t(u, v>0)$ is the beta function, such that

$$
B(u, v)=\frac{1}{\Gamma(u+v)} \Gamma(u) \Gamma(v)
$$

Proof Setting $u=\frac{t}{x}$, we find

$$
\varpi\left(\lambda_{2}, x\right)=x^{\lambda-\lambda_{2}} \int_{0}^{\infty} \frac{(u x)^{\lambda_{2}-1}}{(x+u x)^{\lambda}} x d u=\int_{0}^{\infty} \frac{u^{\lambda_{2}-1}}{(1+u)^{\lambda}} d u=B\left(\lambda_{2}, \lambda-\lambda_{2}\right),
$$

namely, (8) follows. In the same way, we have (9).

The lemma is proved.

Lemma 3 We have the following reverse Hardy-Hilbert integral inequality involving one derivative function:

$$
\begin{aligned}
\int_{0}^{\infty} & \int_{0}^{\infty} \frac{f(x) g^{\prime}(y)}{(x+y)^{\lambda}} d x \\
> & B^{\frac{1}{p}}\left(\lambda_{2}, \lambda-\lambda_{2}\right) B^{\frac{1}{q}}\left(\lambda_{1}, \lambda-\lambda_{1}\right) \\
& \times\left[\int_{0}^{\infty} x^{p\left(1-\lambda_{1}\right)-a-1} f^{p}(x) d x\right]^{\frac{1}{p}}\left[\int_{0}^{\infty} y^{q\left(1-\lambda_{2}\right)-a-1} g^{\prime q}(y) d y\right]^{\frac{1}{q}} .
\end{aligned}
$$

Proof By the reverse Hölder inequality (cf. [30]), we obtain

$$
\begin{aligned}
\int_{0}^{\infty} & \int_{0}^{\infty} \frac{f(x) g^{\prime}(y)}{(x+y)^{\lambda}} d x d y \\
\quad= & \int_{0}^{\infty} \int_{0}^{\infty} \frac{1}{(x+y)^{\lambda}}\left[\frac{y^{\left(\lambda_{2}-1\right) / p}}{x^{\left(\lambda_{1}-1\right) / q}} f(x)\right]\left[\frac{x^{\left(\lambda_{1}-1\right) / q}}{y^{\left(\lambda_{2}-1\right) / p}} g^{\prime}(y)\right] d x d y
\end{aligned}
$$




$$
\begin{aligned}
\geq & \left\{\int_{0}^{\infty}\left[\int_{0}^{\infty} \frac{1}{(x+y)^{\lambda}} \frac{y^{\lambda_{2}-1} d y}{x^{\left(\lambda_{1}-1\right)(p-1)}}\right] f^{p}(x) d x\right\}^{\frac{1}{p}} \\
& \times\left\{\int_{0}^{\infty}\left[\int_{0}^{\infty} \frac{1}{(x+y)^{\lambda}} \frac{x^{\lambda_{1}-1} d x}{y^{\left(\lambda_{2}-1\right)(q-1)}}\right] g^{\prime q}(y) d y\right\}^{\frac{1}{q}} \\
= & {\left[\int_{0}^{\infty} \varpi\left(\lambda_{2}, x\right) x^{p\left(1-\lambda_{1}\right)-a-1} f^{p}(x) d x\right]^{\frac{1}{p}} } \\
& \times\left[\int_{0}^{\infty} \omega\left(\lambda_{1}, y\right) y^{q\left(1-\lambda_{2}\right)-a-1} g^{\prime q}(y) d y\right]^{\frac{1}{q}} .
\end{aligned}
$$

If (12) keeps the form of an equality, then there exist constants $A$ and $B$, such that they are not all zero, satisfying

$$
A \frac{y^{\lambda_{2}-1}}{x^{\left(\lambda_{1}-1\right)(p-1)}} f^{p}(x)=B \frac{x^{\lambda_{1}-1}}{y^{\left(\lambda_{2}-1\right)(q-1)}} g^{\prime q}(y) \quad \text { a.e. in }(0, \infty) \times(0, \infty)
$$

We assume that $A \neq 0$. For fixed a.e. $y \in(0, \infty)$, we have

$$
x^{p\left(1-\lambda_{1}\right)-a-1} f^{p}(x)=\left(\frac{B}{A} y^{q\left(1-\lambda_{2}\right)} g^{\prime q}(y)\right) x^{-1-a} \quad \text { a.e. in }(0, \infty) .
$$

Integration in the above expression, since for any $a=\lambda-\lambda_{1}-\lambda_{2} \in \mathbf{R}, \int_{0}^{\infty} x^{-1-a} d x=\infty$, which contradicts the fact that

$$
0<\int_{0}^{\infty} x^{p\left(1-\lambda_{1}\right)-a-1} f^{p}(x) d x<\infty .
$$

Therefore, by (8) and (9), we have (11).

The lemma is proved.

\section{Main results}

Theorem 1 We have the following reverse Hardy-Hilbert-type integral inequality involving one derivative function:

$$
\begin{aligned}
I:= & \int_{0}^{\infty} \int_{0}^{\infty} \frac{f(x) g(y)}{(x+y)^{\lambda+1}} d x d y \\
> & \frac{1}{\lambda} B^{\frac{1}{p}}\left(\lambda_{2}, \lambda-\lambda_{2}\right) B^{\frac{1}{q}}\left(\lambda_{1}, \lambda-\lambda_{1}\right) \\
& \times\left[\int_{0}^{\infty} x^{p\left(1-\lambda_{1}\right)-a-1} f^{p}(x) d x\right]^{\frac{1}{p}}\left[\int_{0}^{\infty} y^{q\left(1-\lambda_{2}\right)-a-1} g^{\prime q}(y) d y\right]^{\frac{1}{q}} .
\end{aligned}
$$

In particular, for $\lambda_{1}+\lambda_{2}=\lambda$ (or $\left.a=0\right)$, we reduce (13) to the following:

$$
\begin{aligned}
\int_{0}^{\infty} & \int_{0}^{\infty} \frac{f(x) g(y)}{(x+y)^{\lambda+1}} d x d y \\
& >\frac{1}{\lambda} B\left(\lambda_{1}, \lambda_{2}\right)\left(\int_{0}^{\infty} x^{p\left(1-\lambda_{1}\right)-1} f^{p}(x) d x\right)^{\frac{1}{p}}\left(\int_{0}^{\infty} y^{q\left(1-\lambda_{2}\right)-1} g^{\prime q}(y) d y\right)^{\frac{1}{q}}
\end{aligned}
$$

where the constant factor $\frac{1}{\lambda} B\left(\lambda_{1}, \lambda_{2}\right)$ is the best possible. 
Proof Using (4) and (5), in view of the Fubini theorem (cf. [31]), we find

$$
\begin{aligned}
I & =\frac{1}{\Gamma(\lambda+1)} \int_{0}^{\infty} \int_{0}^{\infty} f(x) g(y)\left[\int_{0}^{\infty} t^{(\lambda+1)-1} e^{-(x+y) t} d t\right] d x d y \\
& =\frac{1}{\Gamma(\lambda+1)} \int_{0}^{\infty} t^{(\lambda+1)-1}\left(\int_{0}^{\infty} e^{-x t} f(x) d x\right)\left(\int_{0}^{\infty} e^{-y t} g(y) d y\right) d t \\
& =\frac{1}{\Gamma(\lambda+1)} \int_{0}^{\infty} t^{(\lambda+1)-1}\left(\int_{0}^{\infty} e^{-x t} f(x) d x\right)\left(\int_{0}^{\infty} t^{-1} e^{-y t} g^{\prime}(y) d y\right) d t \\
& =\frac{1}{\lambda \Gamma(\lambda)} \int_{0}^{\infty} \int_{0}^{\infty} f(x) g^{\prime}(y)\left[\int_{0}^{\infty} t^{\lambda-1} e^{-(x+y) t} d t\right] d x d y \\
& =\frac{\Gamma(\lambda)}{\lambda \Gamma(\lambda)} \int_{0}^{\infty} \int_{0}^{\infty} \frac{f(x) g^{\prime}(y)}{(x+y)^{\lambda}} d x d y .
\end{aligned}
$$

Then by (11), we have (13).

For $a=0$ in (13), we have (14). For any $\varepsilon>0$, we set

$$
\tilde{f}(x):=\left\{\begin{array}{ll}
0, & 0<x \leq 1, \\
x^{\lambda_{1}-\frac{\varepsilon}{p}-1}, & x>1,
\end{array} \quad \tilde{g}(y):= \begin{cases}0, & 0<y \leq 1, \\
y^{\lambda_{2}-\frac{\varepsilon}{q}}, & y>1 .\end{cases}\right.
$$

We obtain $\tilde{g}(y)=o(1)\left(y \rightarrow 0^{+}\right), \tilde{g}(y)=o\left(e^{t y}\right)(t>0 ; y \rightarrow \infty), \tilde{g}^{\prime}(y) \equiv 0(0<y<1)$, and

$$
\tilde{g}^{\prime}(y)=\left(\lambda_{2}-\frac{\varepsilon}{q}\right) y^{\lambda_{2}-\frac{\varepsilon}{q}-1} \quad(y>1)
$$

If there exists a constant $M\left(\geq \frac{1}{\lambda} B\left(\lambda_{1}, \lambda_{2}\right)\right)$, such that (14) is valid when replacing $\frac{1}{\lambda} B\left(\lambda_{1}, \lambda_{2}\right)$ by $M$, then in particular, by substitution of $f(x)=\tilde{f}(x), g(y)=\tilde{g}(y)$ and $g^{\prime}(y)=$ $\tilde{g}^{\prime}(y)$, we have

$$
\begin{aligned}
\tilde{I} & :=\int_{0}^{\infty} \int_{0}^{\infty} \frac{\tilde{f}(x) \tilde{g}(y)}{(x+y)^{\lambda+1}} d x d y \\
& >M\left[\int_{0}^{\infty} x^{p\left(1-\lambda_{1}\right)-1} \tilde{f}^{p}(x) d x\right]^{\frac{1}{p}}\left[\int_{0}^{\infty} y^{q\left(1-\lambda_{2}\right)-1} \tilde{g}^{\prime q}(y) d y\right]^{\frac{1}{q}} .
\end{aligned}
$$

We obtain

$$
\begin{aligned}
\tilde{J} & :=\left[\int_{0}^{\infty} x^{p\left(1-\lambda_{1}\right)-1} \tilde{f}^{p}(x) d x\right]^{\frac{1}{p}}\left[\int_{0}^{\infty} y^{q\left(1-\lambda_{2}\right)-1} \tilde{g}^{\prime q}(y) d y\right]^{\frac{1}{q}} \\
& =\left(\lambda_{2}-\frac{\varepsilon}{q}\right)\left(\int_{1}^{\infty} x^{-\varepsilon-1} d x\right)^{\frac{1}{p}}\left(\int_{1}^{\infty} y^{-\varepsilon-1} d y\right)^{\frac{1}{q}} \\
& =\left(\lambda_{2}-\frac{\varepsilon}{q}\right) \int_{1}^{\infty} x^{-\varepsilon-1} d x=\frac{1}{\varepsilon}\left(\lambda_{2}-\frac{\varepsilon}{q}\right) .
\end{aligned}
$$

In view of the Fubini theorem (cf. [31]), it follows that

$$
\tilde{I}=\int_{1}^{\infty}\left[\int_{1}^{\infty} \frac{y^{\lambda_{2}-\frac{\varepsilon}{q}}}{(x+y)^{\lambda+1}} d y\right] x^{\lambda_{1}-\frac{\varepsilon}{p}-1} d x=\int_{1}^{\infty} x^{-\varepsilon-1}\left[\int_{\frac{1}{x}}^{\infty} \frac{u^{\lambda_{2}-\frac{\varepsilon}{q}}}{(1+u)^{\lambda+1}} d u\right] d x
$$




$$
\begin{aligned}
& =\int_{1}^{\infty} x^{-\varepsilon-1}\left[\int_{\frac{1}{x}}^{1} \frac{u^{\lambda_{2}-\frac{\varepsilon}{q}}}{(1+u)^{\lambda+1}} d u\right] d x+\int_{1}^{\infty} x^{-\varepsilon-1}\left[\int_{1}^{\infty} \frac{u^{\lambda_{2}-\frac{\varepsilon}{q}}}{(1+u)^{\lambda+1}} d u\right] d x \\
& =\int_{0}^{1}\left(\int_{\frac{1}{u}}^{\infty} x^{-\varepsilon-1} d x\right) \frac{u^{\lambda_{2}-\frac{\varepsilon}{q}}}{(1+u)^{\lambda+1}} d u+\frac{1}{\varepsilon} \int_{1}^{\infty} \frac{u^{\lambda_{2}-\frac{\varepsilon}{q}}}{(1+u)^{\lambda+1}} d u \\
& =\frac{1}{\varepsilon}\left[\int_{0}^{1} \frac{u^{\lambda_{2}+\frac{\varepsilon}{p}}}{(1+u)^{\lambda+1}} d u+\int_{1}^{\infty} \frac{u^{\lambda_{2}-\frac{\varepsilon}{q}}}{(1+u)^{\lambda+1}} d u\right] .
\end{aligned}
$$

By (16), we obtain

$$
\int_{0}^{1} \frac{u^{\lambda_{2}+\frac{\varepsilon}{p}}}{(1+u)^{\lambda+1}} d u+\int_{1}^{\infty} \frac{u^{\lambda_{2}-\frac{\varepsilon}{q}}}{(1+u)^{\lambda+1}} d u>\varepsilon \tilde{I}>\varepsilon M \tilde{J}>M\left(\lambda_{2}-\frac{\varepsilon}{q}\right) .
$$

Putting $\varepsilon \rightarrow 0^{+}$in the above inequality, in view of the continuity of the beta function, we find

$$
\frac{\lambda_{2}}{\lambda} B\left(\lambda_{1}, \lambda_{2}\right)=\frac{\lambda_{2} \Gamma\left(\lambda_{1}\right) \Gamma\left(\lambda_{2}\right)}{\lambda \Gamma(\lambda)}=B\left(\lambda_{1}, \lambda_{2}+1\right) \geq M \lambda_{2}
$$

namely, $\frac{1}{\lambda} B\left(\lambda_{1}, \lambda_{2}\right) \geq M$. Hence, $M=\frac{1}{\lambda} B\left(\lambda_{1}, \lambda_{2}\right)$ is the best possible constant factor in (14). The theorem is proved.

Remark 1 We set $\hat{\lambda}_{1}:=\lambda_{1}+\frac{a}{p}=\frac{\lambda-\lambda_{2}}{p}+\frac{\lambda_{1}}{q}, \hat{\lambda}_{2}:=\lambda_{2}+\frac{a}{q}=\frac{\lambda-\lambda_{1}}{q}+\frac{\lambda_{2}}{p}$. It follows that $\hat{\lambda}_{1}+\hat{\lambda}_{2}=\lambda$. For

$$
a=\lambda-\lambda_{1}-\lambda_{2} \in\left(-p \lambda_{1}, p\left(\lambda-\lambda_{1}\right)\right)
$$

we find $0<\hat{\lambda}_{1}<\lambda$, and then $0<\hat{\lambda}_{2}=\lambda-\hat{\lambda}_{1}<\lambda$. So we rewrite (13) as follows:

$$
\begin{aligned}
I:= & \int_{0}^{\infty} \int_{0}^{\infty} \frac{f(x) g(y)}{(x+y)^{\lambda+1}} d x d y \\
> & \frac{1}{\lambda} B^{\frac{1}{p}}\left(\lambda_{2}, \lambda-\lambda_{2}\right) B^{\frac{1}{q}}\left(\lambda_{1}, \lambda-\lambda_{1}\right) \\
& \times\left[\int_{0}^{\infty} x^{p\left(1-\hat{\lambda}_{1}\right)-1} f^{p}(x) d x\right]^{\frac{1}{p}}\left[\int_{0}^{\infty} y^{q\left(1-\hat{\lambda}_{2}\right)-1} g^{\prime q}(y) d y\right]^{\frac{1}{q}} .
\end{aligned}
$$

\section{Theorem 2 If the constant factor}

$$
\frac{1}{\lambda} B^{\frac{1}{p}}\left(\lambda_{2}, \lambda-\lambda_{2}\right) B^{\frac{1}{q}}\left(\lambda_{1}, \lambda-\lambda_{1}\right)
$$

in (13) (or (17)) is the best possible, then $\lambda_{1}+\lambda_{2}=\lambda$.

Proof By (14) (for $\left.\lambda_{i}=\hat{\lambda}_{i}(i=1,2)\right)$, since

$$
\frac{1}{\lambda} B^{\frac{1}{p}}\left(\lambda_{2}, \lambda-\lambda_{2}\right) B^{\frac{1}{q}}\left(\lambda_{1}, \lambda-\lambda_{1}\right)
$$

is the best possible constant factor in (17), we have the following inequality:

$$
\frac{1}{\lambda} B^{\frac{1}{p}}\left(\lambda_{2}, \lambda-\lambda_{2}\right) B^{\frac{1}{q}}\left(\lambda_{1}, \lambda-\lambda_{1}\right) \geq \frac{1}{\lambda} B\left(\hat{\lambda}_{1}, \hat{\lambda}_{2}\right) \quad\left(\in \mathrm{R}_{+}\right),
$$


namely,

$$
B\left(\hat{\lambda}_{1}, \hat{\lambda}_{2}\right) \leq B^{\frac{1}{p}}\left(\lambda_{2}, \lambda-\lambda_{2}\right) B^{\frac{1}{q}}\left(\lambda_{1}, \lambda-\lambda_{1}\right) .
$$

By the reverse Hölder inequality (cf. [30]), we obtain

$$
\begin{aligned}
B\left(\hat{\lambda}_{1}, \hat{\lambda}_{2}\right) & =\int_{0}^{\infty} \frac{u^{\hat{\lambda}_{1}-1}}{(1+u)^{\lambda}} d u \\
& =\int_{0}^{\infty} \frac{1}{(1+u)^{\lambda}} u^{\frac{\lambda-\lambda_{2}}{p}+\frac{\lambda_{1}}{q}-1} d u=\int_{0}^{\infty} \frac{1}{(1+u)^{\lambda}}\left(u^{\frac{\lambda-\lambda_{2}-1}{p}}\right)\left(u^{\frac{\lambda_{1}-1}{q}}\right) d u \\
& \geq\left[\int_{0}^{\infty} \frac{u^{\lambda-\lambda_{2}-1}}{(1+u)^{\lambda}} d u\right]^{\frac{1}{p}}\left[\int_{0}^{\infty} \frac{u^{\lambda_{1}-1}}{(1+u)^{\lambda}} d u\right]^{\frac{1}{q}} \\
& =B^{\frac{1}{p}}\left(\lambda_{2}, \lambda-\lambda_{2}\right) B^{\frac{1}{q}}\left(\lambda_{1}, \lambda-\lambda_{1}\right) .
\end{aligned}
$$

It follows that (18) keeps the form of an equality.

We observe that (18) keeps the form of an equality if and only if there exist constants $A$ and $B$, such that they are not all zero and

$$
A u^{\lambda-\lambda_{2}-1}=B u^{\lambda_{1}-1} \quad \text { a.e. in } R_{+}
$$

(cf. [30]). Assuming that $A \neq 0$, it follows that

$$
u^{\lambda-\lambda_{2}-\lambda_{1}}=\frac{B}{A} \quad \text { a.e. in } R_{+} .
$$

We have $a=\lambda-\lambda_{1}-\lambda_{2}=0$, namely, $\lambda_{1}+\lambda_{2}=\lambda$.

The theorem is proved.

Theorem 3 The following statements (i), (ii), (iii) and (iv) are equivalent:

(i) $\operatorname{Both} B^{\frac{1}{p}}\left(\lambda_{2}, \lambda-\lambda_{2}\right) B^{\frac{1}{q}}\left(\lambda_{1}, \lambda-\lambda_{1}\right)$ and $B\left(\frac{\lambda-\lambda_{2}}{p}+\frac{\lambda_{1}}{q}, \frac{\lambda-\lambda_{1}}{q}+\frac{\lambda_{2}}{p}\right)$ are finite and independent of $p, q$;

(ii) $B^{\frac{1}{p}}\left(\lambda_{2}, \lambda-\lambda_{2}\right) B^{\frac{1}{q}}\left(\lambda_{1}, \lambda-\lambda_{1}\right)$ is equal to a single convergent integral

$$
B\left(\hat{\lambda}_{1}, \hat{\lambda}_{2}\right)=\int_{0}^{\infty} \frac{u^{\hat{\lambda}_{1}-1}}{(1+u)^{\lambda}} d u
$$

(iii) if $a=\lambda-\lambda_{1}-\lambda_{2} \in\left(-p \lambda_{1}, p\left(\lambda-\lambda_{1}\right)\right)$, then $\lambda_{1}+\lambda_{2}=\lambda$;

(iv) the constant factor

$$
\frac{1}{\lambda} B^{\frac{1}{p}}\left(\lambda_{2}, \lambda-\lambda_{2}\right) B^{\frac{1}{q}}\left(\lambda_{1}, \lambda-\lambda_{1}\right)
$$

is the best possible in (13).

Proof (i) $\Rightarrow$ (ii). In view of the assumption and the continuity of the beta function, we find

$$
B^{\frac{1}{p}}\left(\lambda_{2}, \lambda-\lambda_{2}\right) B^{\frac{1}{q}}\left(\lambda_{1}, \lambda-\lambda_{1}\right)
$$




$$
\begin{gathered}
=\lim _{p \rightarrow 1^{-}} \lim _{q \rightarrow-\infty} B^{\frac{1}{p}}\left(\lambda_{2}, \lambda-\lambda_{2}\right) B^{\frac{1}{q}}\left(\lambda_{1}, \lambda-\lambda_{1}\right)=B\left(\lambda_{2}, \lambda-\lambda_{2}\right), \\
B\left(\hat{\lambda}_{1}, \hat{\lambda}_{2}\right)=\lim _{p \rightarrow 1^{-}} \lim _{q \rightarrow-\infty} B\left(\frac{\lambda-\lambda_{2}}{p}+\frac{\lambda_{1}}{q}, \frac{\lambda-\lambda_{1}}{q}+\frac{\lambda_{2}}{p}\right)=B\left(\lambda_{2}, \lambda-\lambda_{2}\right) .
\end{gathered}
$$

Hence, $B^{\frac{1}{p}}\left(\lambda_{2}, \lambda-\lambda_{2}\right) B^{\frac{1}{q}}\left(\lambda_{1}, \lambda-\lambda_{1}\right)$ is equal to $B\left(\hat{\lambda}_{1}, \hat{\lambda}_{2}\right)$, which is a single convergent integral.

(ii) $\Rightarrow$ (iii). Suppose that $B^{\frac{1}{p}}\left(\lambda_{2}, \lambda-\lambda_{2}\right) B^{\frac{1}{q}}\left(\lambda_{1}, \lambda-\lambda_{1}\right)$ is equal to a single convergent integral $\int_{0}^{\infty} \frac{1}{(1+u)^{\lambda}} u^{\hat{\lambda}_{1}-1} d u\left(\in \mathrm{R}_{+}\right)$. Then (18) keeps the form of an equality. By the proof of Theorem 2, we have $\lambda_{1}+\lambda_{2}=\lambda$.

(iii) $\Rightarrow$ (iv). If $\lambda_{1}+\lambda_{2}=\lambda$, then by Theorem 1 , the constant factor

$$
\frac{1}{\lambda} B^{\frac{1}{p}}\left(\lambda_{2}, \lambda-\lambda_{2}\right) B^{\frac{1}{q}}\left(\lambda_{1}, \lambda-\lambda_{1}\right)\left(=\frac{1}{\lambda} B\left(\lambda_{1}, \lambda_{2}\right)\right)
$$

in (13) is the best possible.

(iv) $\Rightarrow$ (i). By Theorem 2, we have $\lambda_{1}+\lambda_{2}=\lambda$, and then

$$
\begin{aligned}
& B^{\frac{1}{p}}\left(\lambda_{2}, \lambda-\lambda_{2}\right) B^{\frac{1}{q}}\left(\lambda_{1}, \lambda-\lambda_{1}\right)=B\left(\lambda_{1}, \lambda_{2}\right), \\
& B\left(\frac{\lambda-\lambda_{2}}{p}+\frac{\lambda_{1}}{q}, \frac{\lambda-\lambda_{1}}{q}+\frac{\lambda_{2}}{p}\right)=B\left(\lambda_{1}, \lambda_{2}\right) .
\end{aligned}
$$

It follows that both of them are finite and independent of $p, q$.

Hence, the statements (i), (ii), (iii) and (iv) are equivalent.

The theorem is proved.

Remark 2 For $a=0$ in (11), we have

$$
\begin{aligned}
& \int_{0}^{\infty} \int_{0}^{\infty} \frac{f(x) g^{\prime}(y)}{(x+y)^{\lambda}} d x \\
& \quad>B\left(\lambda_{1}, \lambda_{2}\right)\left[\int_{0}^{\infty} x^{p\left(1-\lambda_{1}\right)-1} f^{p}(x) d x\right]^{\frac{1}{p}}\left[\int_{0}^{\infty} y^{q\left(1-\lambda_{2}\right)-1} g^{\prime q}(y) d y\right]^{\frac{1}{q}} .
\end{aligned}
$$

We conform that the constant factor $B\left(\lambda_{1}, \lambda_{2}\right)$ in (19) is the best possible. Otherwise, we would reach a contradiction by (15) (for $a=0$ ): the constant factor in (14) is not the best possible.

\section{Equivalent form and some particular inequalities}

Theorem 4 Inequality (13) is equivalent to the following reverse Hardy-Hilbert-type integral inequality involving one derivative function:

$$
\begin{aligned}
J: & =\left\{\int_{0}^{\infty} x^{q\left(\lambda_{1}+a\right)-a-1}\left[\int_{0}^{\infty} \frac{g(y)}{(x+y)^{\lambda+1}} d y\right]^{q} d x\right\}^{\frac{1}{q}} \\
& >\frac{1}{\lambda} B^{\frac{1}{p}}\left(\lambda_{2}, \lambda-\lambda_{2}\right) B^{\frac{1}{q}}\left(\lambda_{1}, \lambda-\lambda_{1}\right)\left[\int_{0}^{\infty} y^{q\left(1-\lambda_{2}\right)-a-1} g^{\prime q}(y) d y\right]^{\frac{1}{q}} .
\end{aligned}
$$


In particular, for $\lambda_{1}+\lambda_{2}=\lambda$ (or $\left.a=0\right)$, we reduce (20) to the equivalent form of (14) as follows:

$$
\left\{\int_{0}^{\infty} x^{q \lambda_{1}-1}\left[\int_{0}^{\infty} \frac{g(y)}{(x+y)^{\lambda+1}} d y\right]^{q} d x\right\}^{\frac{1}{q}}>\frac{1}{\lambda} B\left(\lambda_{1}, \lambda_{2}\right)\left[\int_{0}^{\infty} y^{q\left(1-\lambda_{2}\right)-1} g^{\prime q}(y) d y\right]^{\frac{1}{q}}
$$

where the constant factor $\frac{1}{\lambda} B\left(\lambda_{1}, \lambda_{2}\right)$ is the best possible.

Proof Suppose that (20) is valid. By the reverse Hölder integral inequality (cf. [30]), we have

$$
\begin{aligned}
I & =\int_{0}^{\infty}\left[x^{\frac{1}{q}-\lambda_{1}-\frac{a}{p}} f(x)\right]\left[x^{-\frac{1}{q}+\lambda_{1}+\frac{a}{p}} \int_{0}^{\infty} \frac{g(y)}{(x+y)^{\lambda+1}} d y\right] d x \\
& \geq\left\{\int_{0}^{\infty} x^{p\left(1-\lambda_{1}\right)-a-1} f^{p}(x) d x\right\}^{\frac{1}{p}} J .
\end{aligned}
$$

Then by (20), we have (13).

On the other hand, assuming that (13) is valid, we set

$$
f(x):=x^{q\left(\lambda_{1}+a\right)-a-1}\left[\int_{0}^{\infty} \frac{g(y)}{(x+y)^{\lambda+1}} d y\right]^{q-1}, \quad x \in \mathrm{R}_{+} .
$$

If $J=\infty$, then (20) is naturally valid; if $J=0$, then it is impossible to make (20) valid, namely $J>0$. Suppose that $0<J<\infty$. By (13), we have

$$
\begin{aligned}
& \int_{0}^{\infty} x^{p\left(1-\lambda_{1}\right)-a-1} f^{p}(x) d x \\
& =J^{q}=I>\frac{1}{\lambda} B^{\frac{1}{p}}\left(\lambda_{2}, \lambda-\lambda_{2}\right) B^{\frac{1}{q}}\left(\lambda_{1}, \lambda-\lambda_{1}\right) J^{q-1}\left[\int_{0}^{\infty} y^{q\left(1-\lambda_{2}\right)-a-1} g^{\prime q}(y) d y\right]^{\frac{1}{q}}, \\
& J=\left[\int_{0}^{\infty} x^{p\left(1-\lambda_{1}\right)-a-1} f^{p}(x) d x\right]^{\frac{1}{q}} \\
& >\frac{1}{\lambda} B^{\frac{1}{p}}\left(\lambda_{2}, \lambda-\lambda_{2}\right) B^{\frac{1}{q}}\left(\lambda_{1}, \lambda-\lambda_{1}\right)\left[\int_{0}^{\infty} y^{q\left(1-\lambda_{2}\right)-a-1} g^{\prime q}(y) d y\right]^{\frac{1}{q}},
\end{aligned}
$$

namely, (20) follows, which is equivalent to (13).

The constant factor $\frac{1}{\lambda} B\left(\lambda_{1}, \lambda_{2}\right)$ is the best possible in (21). Otherwise, by (22) (for $a=0$ ), we would reach a contradiction: that the constant factor in (14) is not the best possible.

The theorem is proved.

Replacing $x$ by $\frac{1}{x}$, and then replacing $x^{\lambda-1} f\left(\frac{1}{x}\right)$ by $f(x)$ in (13) and (20), by calculation, we have the following.

Corollary 1 The following reverse Hardy-Hilbert-type integral inequalities with the nonhomogeneous kernel involving one derivative function are equivalent:

$$
\int_{0}^{\infty} \int_{0}^{\infty} \frac{f(x) g(y)}{(1+x y)^{\lambda+1}} d x d y
$$




$$
\begin{aligned}
&> \frac{1}{\lambda} B^{\frac{1}{p}}\left(\lambda_{2}, \lambda-\lambda_{2}\right) B^{\frac{1}{q}}\left(\lambda_{1}, \lambda-\lambda_{1}\right) \\
& \times\left[\int_{0}^{\infty} x^{p\left(\lambda_{1}-\lambda\right)+a-1} f^{p}(x) d x\right]^{\frac{1}{p}}\left[\int_{0}^{\infty} y^{q\left(1-\lambda_{2}\right)-a-1} g^{\prime q}(y) d y\right]^{\frac{1}{q}}, \\
&\left\{\int_{0}^{\infty} x^{q\left(\lambda-\lambda_{1}-a+1\right)+a-1}\left[\int_{0}^{\infty} \frac{g(y)}{(1+x y)^{\lambda+1}} d y\right]^{q} d x\right\}^{\frac{1}{q}} \\
&>\frac{1}{\lambda} B^{\frac{1}{p}}\left(\lambda_{2}, \lambda-\lambda_{2}\right) B^{\frac{1}{q}}\left(\lambda_{1}, \lambda-\lambda_{1}\right)\left[\int_{0}^{\infty} y^{q\left(1-\lambda_{2}\right)-a-1} g^{\prime q}(y) d y\right]^{\frac{1}{q}} .
\end{aligned}
$$

Moreover, $\lambda_{1}+\lambda_{2}=\lambda$ (or $\left.a=0\right)$ if and only if the constant factor $\frac{1}{\lambda} B^{\frac{1}{p}}\left(\lambda_{2}, \lambda-\lambda_{2}\right) B^{\frac{1}{q}}\left(\lambda_{1}, \lambda-\right.$ $\left.\lambda_{1}\right)$ in (23) and (24) is the best possible.

For $\lambda_{1}+\lambda_{2}=\lambda$ (or $a=0$ ), we have the following reverse equivalent inequalities with the non-homogeneous kernel and the best possible constant factor $\frac{1}{\lambda} B\left(\lambda_{1}, \lambda_{2}\right)$ :

$$
\begin{aligned}
& \int_{0}^{\infty} \int_{0}^{\infty} \frac{f(x) g(y)}{(1+x y)^{\lambda+1}} d x d y \\
& >\frac{1}{\lambda} B\left(\lambda_{1}, \lambda_{2}\right) \times\left(\int_{0}^{\infty} x^{-p \lambda_{2}-1} f^{p}(x) d x\right)^{\frac{1}{p}}\left[\int_{0}^{\infty} y^{q\left(1-\lambda_{2}\right)-1} g^{\prime q}(y) d y\right]^{\frac{1}{q}} \\
& \left\{\int_{0}^{\infty} x^{q\left(\lambda_{2}+1\right)-1}\left[\int_{0}^{\infty} \frac{g(y)}{(1+x y)^{\lambda+1}} d y\right]^{q} d x\right\}^{\frac{1}{q}} \\
& >\frac{1}{\lambda} B\left(\lambda_{1}, \lambda_{2}\right)\left[\int_{0}^{\infty} y^{q\left(1-\lambda_{2}\right)-1} g^{\prime q}(y) d y\right]^{\frac{1}{q}}
\end{aligned}
$$

Remark 3 For $\lambda_{1}=\frac{\lambda}{r}, \lambda_{2}=\frac{\lambda}{s}\left(r>1, \frac{1}{r}+\frac{1}{s}=1\right)$ in (14), (21), (25) and (26), we have the following two couples of reverse equivalent integral inequalities with the same best possible constant factor $\frac{1}{\lambda} B\left(\frac{\lambda}{r}, \frac{\lambda}{s}\right)$ :

$$
\begin{aligned}
& \int_{0}^{\infty} \int_{0}^{\infty} \frac{f(x) g(y)}{(x+y)^{\lambda+1} d x d y} \\
& >\frac{1}{\lambda} B\left(\frac{\lambda}{r}, \frac{\lambda}{s}\right)\left[\int_{0}^{\infty} x^{p\left(1-\frac{\lambda}{r}\right)-1} f^{p}(x) d x\right]^{\frac{1}{p}}\left[\int_{0}^{\infty} y^{q\left(1-\frac{\lambda}{s}\right)-1} g^{\prime q}(y) d y\right]^{\frac{1}{q}} \\
& \left\{\int_{0}^{\infty} x^{\frac{q \lambda}{r}-1}\left[\int_{0}^{\infty} \frac{g(y)}{(x+y)^{\lambda+1}} d y\right]^{q} d x\right\}^{\frac{1}{q}} \\
& >\frac{1}{\lambda} B\left(\frac{\lambda}{r}, \frac{\lambda}{s}\right)\left[\int_{0}^{\infty} y^{q\left(1-\frac{\lambda}{s}\right)-1} g^{\prime q}(y) d y\right]^{\frac{1}{q}} \\
& \int_{0}^{\infty} \int_{0}^{\infty} \frac{f(x) g(y)}{(1+x y)^{\lambda+1}} d x d y \\
& >\frac{1}{\lambda} B\left(\frac{\lambda}{r}, \frac{\lambda}{s}\right)\left(\int_{0}^{\infty} x^{-\frac{p \lambda}{s}-1} f^{p}(x) d x\right)^{\frac{1}{p}}\left[\int_{0}^{\infty} y^{q\left(1-\frac{\lambda}{s}\right)-1} g^{\prime q}(y) d y\right]^{\frac{1}{q}} \\
& \left\{\int_{0}^{\infty} x^{q\left(\frac{\lambda}{s}+1\right)-1}\left[\int_{0}^{\infty} \frac{g(y)}{(1+x y)^{\lambda+1}} d y\right]^{q} d x\right\}^{\frac{1}{q}} \\
& >\frac{1}{\lambda} B\left(\frac{\lambda}{r}, \frac{\lambda}{s}\right)\left[\int_{0}^{\infty} y^{q\left(1-\frac{\lambda}{s}\right)-1} g^{\prime q}(y) d y\right]^{\frac{1}{q}}
\end{aligned}
$$


In particular, for $\lambda=1, r=s=2$, we have

$$
\begin{aligned}
& \int_{0}^{\infty} \int_{0}^{\infty} \frac{f(x) g(y)}{(x+y)^{2}} d x d y>\pi\left(\int_{0}^{\infty} x^{\frac{p}{2}-1} f^{p}(x) d x\right)^{\frac{1}{p}}\left(\int_{0}^{\infty} y^{\frac{q}{2}-1} g^{\prime q}(y) d y\right)^{\frac{1}{q}}, \\
& \left\{\int_{0}^{\infty} x^{\frac{q}{2}-1}\left[\int_{0}^{\infty} \frac{g(y)}{(x+y)^{2}} d y\right]^{q} d x\right\}^{\frac{1}{q}}>\pi\left(\int_{0}^{\infty} y^{\frac{q}{2}-1} g^{\prime q}(y) d y\right)^{\frac{1}{q}} \\
& \int_{0}^{\infty} \int_{0}^{\infty} \frac{f(x) g(y)}{(1+x y)^{2}} d x d y>\pi\left(\int_{0}^{\infty} x^{-\frac{p}{2}-1} f^{p}(x) d x\right)^{\frac{1}{p}}\left(\int_{0}^{\infty} y^{\frac{q}{2}-1} g^{\prime q}(y) d y\right)^{\frac{1}{q}}, \\
& \left\{\int_{0}^{\infty} x^{\frac{3 q}{2}-1}\left[\int_{0}^{\infty} \frac{g(y)}{(1+x y)^{2}} d y\right]^{q} d x\right\}^{\frac{1}{q}}>\pi\left(\int_{0}^{\infty} y^{\frac{q}{2}-1} g^{\prime q}(y) d y\right)^{\frac{1}{q}} .
\end{aligned}
$$

\section{Conclusions}

In this paper, following [21, 23], by the use of weight functions, the idea of introducing parameters, the reverse extension of (1) and the technique of real analysis, a reverse Hardy-Hilbert-type integral inequality with the kernel $\frac{1}{(x+y)^{\lambda+1}}(\lambda>0)$ involving one derivative function and the beta function is given in Theorem 1 . The equivalent statements of the best possible constant factor related to several parameters are considered in Theorem 3 . The equivalent form, the cases of non-homogeneous kernel and a few particular inequalities are obtained in Theorem 4, Corollary 1 and Remark 3. The lemmas and theorems provide an extensive account of this type of inequalities.

\section{Acknowledgements}

The authors thank the referee for a useful proposal to reform the paper.

\section{Funding}

This work is supported by the National Natural Science Foundation (No. 61772140), and Science and Technology Planning Project Item of Guangzhou City (No. 201707010229). We are grateful for their help.

\section{Availability of data and materials}

We declare that the data and material in this paper can be used publicly.

\section{Competing interests}

The authors declare that they have no competing interests.

Authors' contributions

BY carried out the mathematical studies, participated in the sequence alignment and drafted the manuscript. QC participated in the design of the study and performed the numerical analysis. All authors read and approved the final manuscript.

\section{Author details}

${ }^{1}$ Department of Computer Science, Guangdong University of Education, Guangzhou, Guangdong 51003, China.

${ }^{2}$ Department of Mathematics, Guangdong University of Education, Guangzhou, Guangdong 51003, China.

\section{Publisher's Note}

Springer Nature remains neutral with regard to jurisdictional claims in published maps and institutional affiliations.

Received: 20 March 2020 Accepted: 7 December 2020 Published online: 11 December 2020

\section{References}

1. Hardy, G.H., Littlewood, J.E., Polya, G.: Inequalities. Cambridge University Press, Cambridge (1934)

2. Yang, B.C.: The Norm of Operator and Hilbert-Type Inequalities. Science Press, Beijing (2009)

3. Yang, B.C.: Hilbert-Type Integral Inequalities. Bentham Science Publishers, The United Arab Emirates (2009)

4. Yang, B.C.: On the norm of an integral operator and applications. J. Math. Anal. Appl. 321, 182-192 (2006)

5. Xu, J.S.: Hardy-Hillbert's inequalities with two parameters. Adv. Math. 36(2), 63-76 (2007)

6. Yang, B.C.: On the norm of a Hilbert's type linear operator and applications. J. Math. Anal. Appl. 325, 529-541 (2007)

7. Xie, Z.T., Zeng, Z., Sun, Y.F.: A new Hilbert-type inequality with the homogeneous kernel of degree -2. Adv. Appl. Math. Sci. 12(7), 391-401 (2013) 
8. Zhen, Z., Raja Rama Gandhi, K., Xie, Z.T.: A new Hilbert-type inequality with the homogeneous kernel of degree -2 and with the integral. Bull. Math. Sci. Appl. 3(1), 11-20 (2014)

9. Xin, D.M.: A Hilbert-type integral inequality with the homogeneous kernel of zero degree. Math. Theory Appl. 30(2), 70-74 (2010)

10. Azar, L.E.: The connection between Hilbert and Hardy inequalities. Arch. Inequal. Appl. 2013, 452 (2013)

11. Batbold, T., Sawano, Y: Sharp bounds for m-linear Hilbert-type operators on the weighted Morrey spaces. Math. Inequal. Appl. 20, 263-283 (2017)

12. Adiyasuren, V., Batbold, T., Krnic, M.: Multiple Hilbert-type inequalities involving some differential operators. Banach J. Math. Anal. 10, 320-337 (2016)

13. Adiyasuren, V., Batbold, T., Krni 'c, M.: Hilbert-type inequalities involving differential operators, the best constants and applications. Math. Inequal. Appl. 18, 111-124 (2015)

14. Rassias, M.T., Yang, B.C.: On half-discrete Hilbert's inequality. Appl. Math. Comput. 220, 75-93 (2013)

15. Yang, B.C., Krnic, M.: A half-discrete Hilbert-type inequality with a general homogeneous kernel of degree 0. J. Math. Inequal. 6(3), 401-417 (2012)

16. Rassias, M.T., Yang, B.C.: A multidimensional half-discrete Hilbert-type inequality and the Riemann zeta function. Appl. Math. Comput. 225, 263-277 (2013)

17. Rassias, M.T., Yang, B.C.: On a multidimensional half-discrete Hilbert-type inequality related to the hyperbolic cotangent function. Appl. Math. Comput. 242, 800-813 (2013)

18. Huang, Z.X., Yang, B.C.: On a half-discrete Hilbert-type inequality similar to Mulholland's inequality. Arch. Inequal. Appl. 2013, 290 (2013)

19. Yang, B.C., Lebnath, L.: Half-Discrete Hilbert-Type Inequalities. World Scientific, Singapore (2014)

20. Krnic, M., Pecaric, J.: Extension of Hilbert's inequality. J. Math. Anal. Appl. 324(1), 150-160 (2006)

21. Adiyasuren, V., Batbold, T., Azar, L.E.: A new discrete Hilbert-type inequality involving partial sums. Arch. Inequal. Appl. 2019, $127(2019)$

22. Mo, H.M., Yang, B.C.: On a new Hilbert-type integral inequality involving the upper limit functions. Arch. Inequal. Appl. $2020,5(2020)$

23. Hong, Y., Wen, Y.M.: A necessary and sufficient condition of that Hilbert type series inequality with homogeneous kernel has the best constant factor. Ann. Math. 37A(3), 329-336 (2016)

24. Hong, Y:: On the structure character of Hilbert's type integral inequality with homogeneous kernel and applications. J. Jilin Univ. Sci. Ed. 55(2), 189-194 (2017)

25. Hong, Y., Huang, Q.L., Yang, B.C., Liao, J.L.: The necessary and sufficient conditions for the existence of a kind of Hilbert-type multiple integral inequality with the non-homogeneous kernel and its applications. Arch. Inequal. Appl. 2017, $316(2017)$

26. Xin, D.M., Yang, B.C., Wang, A.Z.: Equivalent property of a Hilbert-type integral inequality related to the beta function in the whole plane. J. Funct. Spaces 2018, Article ID 2691816 (2018)

27. Hong, Y., He, B., Yang, B.C.: Necessary and sufficient conditions for the validity of Hilbert type integral inequalities with a class of quasi-homogeneous kernels and its application in operator theory. J. Math. Inequal. 12(3), 777-788 (2018)

28. Liao, J.Q., Wu, S.H., Yang, B.C.: On a new half-discrete Hilbert-type inequality involving the variable upper limit integral and the partial sum. Mathematics 8, 229 (2020). https://doi.org/10.3390/math8020229

29. Wang, Z.X., Guo, D.R.: Introduction to Special Functions. Science Press, Beijing (1979)

30. Kuang, J.C.: Applied Inequalities. Shangdong Science and Technology Press, Jinan (2004)

31. Kuang, J.C.: Real and Functional Analysis (Continuation), vol. 2. Higher Education Press, Beijing (2015)

\section{Submit your manuscript to a SpringerOpen ${ }^{\circ}$ journal and benefit from:}

- Convenient online submission

- Rigorous peer review

- Open access: articles freely available online

- High visibility within the field

- Retaining the copyright to your article

Submit your next manuscript at $\boldsymbol{\nabla}$ springeropen.com 